\title{
SCIENTIfic Poster Presentation Abstracts
}

Table 1. Statistical Comparison of Kalman Filter and the Conventional Method by t-test; $\mathrm{h}$ is the Decision Based on t-test, 0 Means that Zero Hypothesis is Not Rejected and 1 Means That Alternative Hypothesis is Significant

\begin{tabular}{lcc}
\hline t-test & h & P Value \\
\hline Kalman Filter vs. True values & 0 & 0.2440 \\
\hline Conventional method vs. True values & 1 & $1.8563 \mathrm{e}-48$ \\
\hline Conventional method vs. Kalman filter & 1 & $2.5404 \mathrm{e}-33$ \\
\hline
\end{tabular}

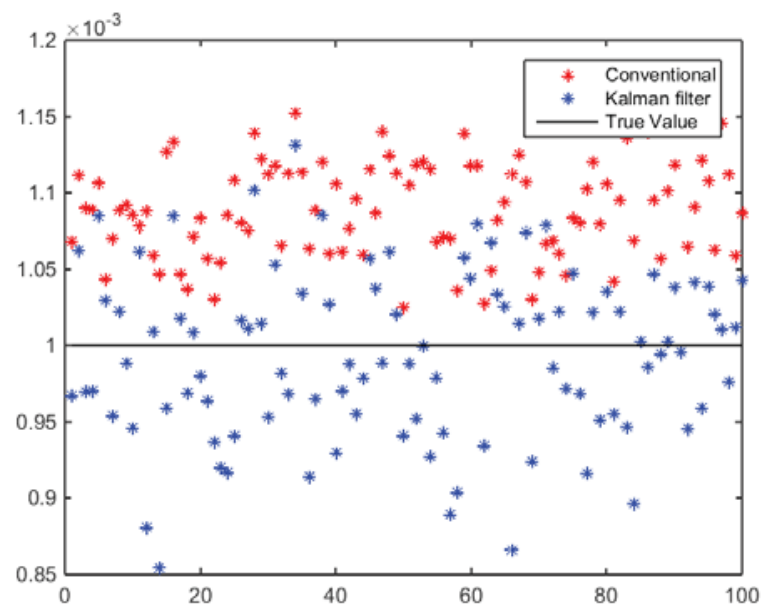

Figure 2. Comparison of the results of Kalman-Filter and conventional method to calculate D. As can be seen, D in the conventional method has a bias.

\section{Bibliography}

1. A. R. Padhani, G. Liu, D. Mu-Koh, T. L. Chenevert, H. C. Thoeny, T. Takahara, A. Dzik-Jurasz, B. D. Ross, M. V. Cauteren, D. Collins, D. A. Hammoud, G. J. Rustin, B. Taouli and P. L. Choyke, "Diffusion-Weighted Magnetic Resonance Imaging as a Cancer Biomarker: Consensus and Recommendations," Neoplasia, vol. 11, no. 2, pp. 102-125, 2009.

2. E. O. Stejskal and J. E. Tanner, "Spin Diffusion Measurements: Spin Echoes in the Presence of a Time-Dependent Field Gradient," Journal of Chemical Physics, vol. 42, no. 1, pp. 288-292, 1965.

\section{-10.5812/IRANJRADIOL.99154}

\section{A Diagnostic Machine Classifier Using Multi-Parametric MRI to Differentiate Benign from Malignant Myometrial Tumors}

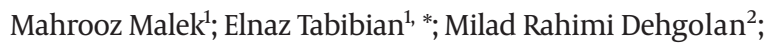

Maryam Rahmani'; Setareh Akhavan'; Shahrzad Sheikh Hasani ${ }^{1}$; Fatemeh Nili ${ }^{1}$; Hassan Hashemi ${ }^{1}$

${ }^{1}$ Tehran University of Medical Sciences, Tehran, Iran

${ }^{2}$ Khaje Nasir Toosi University of Technology, Tehran, Iran

${ }^{*}$ Corresponding author:Tehran University of Medical Sciences, Tehran, Iran.Email: elnaz.tabibian@gmail.com

\section{Abstract}

Background: There are many conditions in medicine that decision making has crucial importance to differentiate between binary diagnoses, such as preoperative discrimination of benign from malignant tumors, e.g. uterine neoplasms. Physicians are not usually able to pool multiple parameters affecting the diagnosis, while "machine learning" techniques, especially "decision trees" with humanreadable results, can process such amounts of data. Previous studies have shown that MRI could be helpful in the differentiation of uterine leiomyosarcoma from leiomyoma.

Objectives: Hereby, for preoperative diagnosis of these tumors and to reduce unnecessary costs and surgeries, we applied a machine classifier using multi-parametric MRI to construct practical diagnostic algorithms.

Methods: A total of 105 myometrial lesions were included in two groups of benign and malignant, according to postoperative tissue diagnosis. Multi-parametric MRI including T1-, T2-, and diffusion-weighted images (DWI) with apparent diffusion coefficient (ADC) map, contrast-enhanced images, as well as MR spectroscopy, were performed for each lesion. Thirteen singular MRI features were extracted from the mentioned sequences. Various combination sets of selective features were fed into a machine classifier (coarse decision-tree) to predict malignant or benign tumors. The accuracy metrics of either singular or combinational models were assessed (Figures 1 and 2). Eventually, two diagnostic algorithms, including a simple decision-tree and a complex one, were proposed using the most accurate models by MATLAB 2017.

Results: Among all singular features, the visual assessment of DWI-ADC restriction, followed by the T2 map, achieved the best accuracies as $96.2 \%$ and $92.0 \%$, respectively. Our final simple decision-tree comprised three features including T2, central necrosis (CN), and DWI-ADC restriction with accuracy of $96.2 \%$, sensitivity of $100 \%$, and specificity of $95 \%$. 


\section{SCientific Poster Presentation Abstracts}

However, the complex tree made up of four features including T2 map, CN, DWI-ADC restriction, and Tumor to Myometrium Contrast-Enhanced Ratio yielded accuracy, sensitivity, and specificity values of $100 \%$.

Conclusion: The complex diagnostic algorithm, compared to the simple model, could differentiate tumors, with equal sensitivity but higher specific- ity. However, it needs more advanced calculations and a high level of patient cooperation; moreover, it might be a time-consuming method. Therefore, physicians should wisely trade-off in different clinical situations and request imaging modalities in a way to reduce additional costs and most importantly, to prevent unnecessary surgeries, by helping early accurate diagnosis.
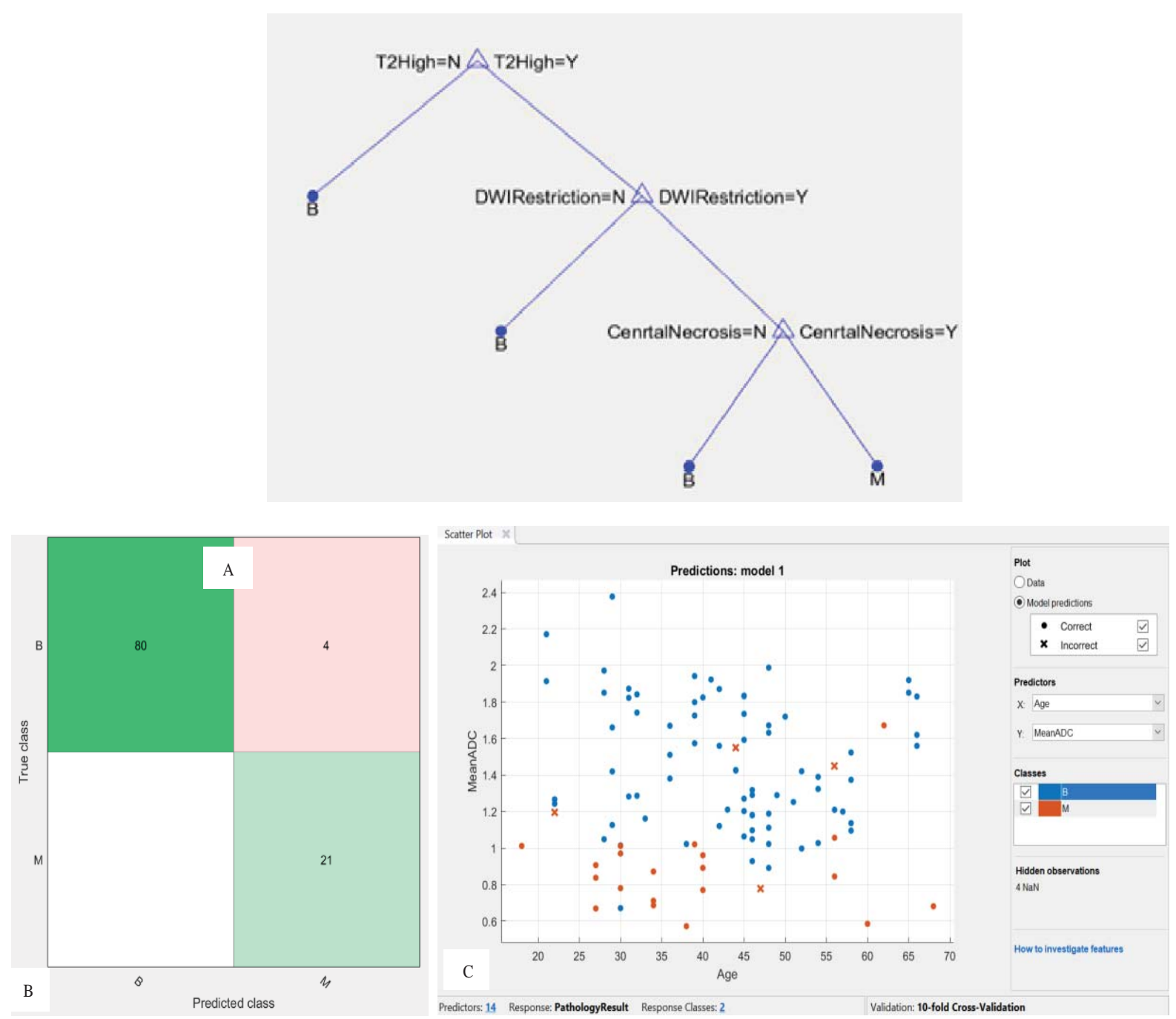

Figure 1. A, simple decision tree using three parameters of predominant T2 signal, DWI restriction, and central necrosis; B, confusion matrix for the number of lesions on true and false predicted classes; C, scatter-plot for distribution of lesions based on the simple model. B, Benign; M, malignant; $\mathrm{Y}$, yes; N, no. 


\section{SCIENTIFIC Poster Presentation AbStraCtS}

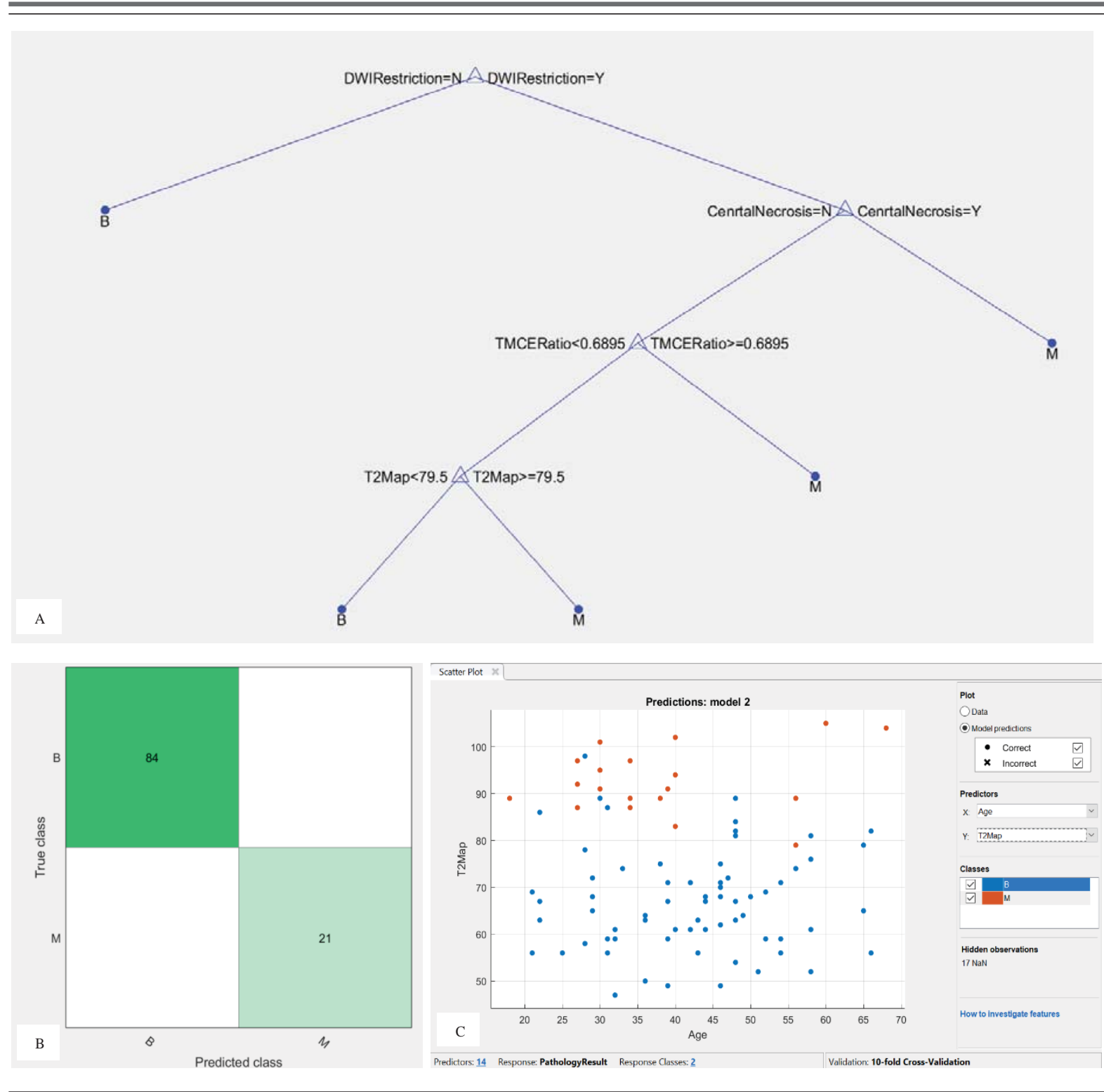

Figure 2. A, complex decision tree using four parameters of DWI restriction, central necrosis, T2 map, and TMCE ratio; B, confusion matrix for the number of lesions on true and false predicted classes; C, scatter-plot for distribution of lesions based on the complex model. B, Benign; M, malignant; Y, yes; N, no.

Keywords: Diagnostic Algorithms; Machine Learning; Leiomyosarcoma; Decision Trees; Uterine Neoplasms

\section{-10.5812/IRANJRADIOL.99155}

\section{Evaluation of IoT Capability in Detecting Kidney Malformations on Ultrasound Imaging System}

Ali Hajipourtalebi ${ }^{1}$; Monireh Tahvildarzadeh ${ }^{2}$; Soheila Artificial Intelligence in Medical Imaging. 2019 November
Vashghani Farahani ${ }^{3,}$; Mehrangiz Ghabimi ${ }^{4}$; Sadegh Taheri $^{1}$

${ }^{1}$ Student Research Committee, Faculty of Nursing, AJA University of Medical Sciences, Tehran, Iran

${ }^{2}$ Health Information Technology Group, Lorestan University of Medical Sciences, Khoramabad, Iran

${ }^{3}$ Student Research Committee, Lorestan University of Medical Sciences, Khoramabad, Iran

${ }^{4}$ Zahedan University of Medical Sciences, Zahedan, Iran

${ }^{*}$ Corresponding author: B.Sc. Student of Health Information Technology, Student Research Committee, Lorestan University of Medical Sciences, Khoramabad, Iran.Email: savasheghanifarahani@gmail.com 